\title{
Dynamics of a rope modeled as a multi-body system with elastic joints
}

\author{
Paweł Fritzkowski · Henryk Kamiński
}

Received: 27 April 2009 / Accepted: 25 July 2010 / Published online: 10 August 2010

(C) The Author(s) 2010. This article is published with open access at Springerlink.com

\begin{abstract}
A discrete model of a rope with spiral springs in joints is considered, the aim being to include transverse elasticity of the rope. Elastic characteristic of the springs is derived on the basis of simple geometrical formulas and the classical curvature-bending moment relationship for beams. Lagrange's equations of motion are presented and their complexity is discussed from the computational point of view. Numerical experiments are performed for a system with both scleronomic and rheonomic constraints. The influence of the elasticity on behaviour of the model is analyzed. Results validity is examined in terms of basic energy principles.
\end{abstract}

Keywords Ropes · Discrete model $\cdot$ Multi-body dynamics · Transverse elasticity

\section{Introduction}

Among classical problems of mechanics, one may find dynamics of a rope as still interesting and non-trivial. As with closely related motion of chains, whips or flylines, behaviour of the rope is imaginable, intuitively comprehended, however, performing numerical simulations in this area is definitely a complex and challenging task. Generally speaking, the problem solving requires an efficient combining modeling techniques with computational methods.

In recent years, dynamics of the mentioned bodies has been widely considered, also with use of numerical experiments. In $[1,2]$ one may find analysis of motion of a folded

P. Fritzkowski $(\bowtie) \cdot$ H. Kamiński

Institute of Applied Mechanics, Poznań University of Technology,

Piotrowo 3, 60-965 Poznan, Poland

e-mail: pawel.fritzkowski@gmail.com

H. Kamiński

e-mail: henryk.kaminski@put.poznan.pl chain. Gorielly and McMillen [3] deal with a whip cracking phenomenon. The work [4], in turn, is devoted to dynamics of a flyline. Since the present paper develops a multi-body system, the reader is referred especially to $[5,6]$, where authors discuss models of inverted multiple pendulums with various elastic characteristics of springs in joints.

In [7] we studied dynamics of a rope modeled as a multiple physical pendulum. This approach has led to an expanded set of differential equations, and, more importantly, the simulated behaviour was found to become disordered, chaoticlooking and therefore quite far from the intuitive view on the rope dynamics. In [8] we modified the simple model to include longitudinal elasticity. Now, we focus on transverse elasticity of the rope. More precisely, the multi-body system consisting of rigid elements is modified by mean of spiral springs placed in its joints. An implementation of this idea is expected to make the mechanical system more realistic, particularly by eliminating the unnatural, individual rotations of the members described in [7].

In what follows we present Lagrange equations of motion of the discussed system, considering rheonomic constraints in general. Next, we turn to a numerical model of the problem and explain its complexity from the computational point of view. Initial value problems, formulated in the presented examples, are solved numerically. On the basis of the performed simulations, we analyze behaviour of the body, concentrating mostly on the elasticity and its impact on the dynamics.

\section{Mechanical system and equations of motion}

As mentioned above, the simplest, preliminary model of a rope is specified in [7]. Since it lays the foundations for the present paper, let us recall the model assumptions. Generally, 
we consider a rope placed in a gravitational field where no external forces are present. One end of the body is attached to a point $O\left(x_{0}, y_{0}\right)$, whereas the other one is released. Classically, the motion is restricted to take place in a vertical plane only. The rope is modeled as a discrete system which consists of $n$ identical members, being rigid prismatic rods of a length $l$ and mass $m$. They are connected by ideal (frictionless) joints.

Let us show now the mathematical description briefly, for more details the reader is referred to [7]. To make the description more general, we have focused on the system with rheonomic constraints by assuming that the point $O$ may be a moving support, whose position is explicitly time-dependent

$x_{0}=x_{0}(t), \quad y_{0}=y_{0}(t)$

Having chosen the angular generalized coordinates

$\mathbf{q}=\left[\varphi_{1}, \varphi_{2}, \ldots, \varphi_{n}\right]^{T}$,

which specify orientation of each element, one may write the set of transformation equations for $i=1,2, \ldots, n$ :

$x_{i}=x_{0}+\sum_{j=1}^{i-1} l \sin \varphi_{j}+\frac{1}{2} l \sin \varphi_{i}$

$y_{i}=y_{0}+\sum_{j=1}^{i-1} l \cos \varphi_{j}+\frac{1}{2} l \cos \varphi_{i}$

where $x_{i}$ and $y_{i}$ are rectangular coordinates of the mass center of the $i$ th body segment. Using the terms (1) and their time derivatives, we can determine the kinetic and the potential energy of the system, $T$ and $V$ respectively. Denoting the Lagrangian by $L=T-V$ one may write the equations of motion in the general form:

$\frac{d}{d t}\left(\frac{\partial L}{\partial \dot{\varphi}_{i}}\right)-\frac{\partial L}{\partial \varphi_{i}}=0, \quad i=1,2, \ldots, n$

For the given system, after substitutions and simplifications the Lagrange equations become

$$
\begin{aligned}
& \sum_{j=1}^{n} a_{i j} \ddot{\varphi}_{j} \cos \left(\varphi_{i}-\varphi_{j}\right)+\sum_{j=1}^{n} a_{i j} \dot{\varphi}_{j}^{2} \sin \left(\varphi_{i}-\varphi_{j}\right) \\
& \quad+\frac{b_{i}}{l}\left(g \sin \varphi_{i}+\ddot{x}_{0} \cos \varphi_{i}-\ddot{y}_{0} \sin \varphi_{i}\right)=0, \quad i=1,2, \ldots, n
\end{aligned}
$$

where

$$
\begin{aligned}
& a_{i j}= \begin{cases}\frac{2(n-i)+1}{2} & \text { for } j<i \\
\frac{3(n-i)+1}{3} & \text { for } j=i \\
\frac{2(n-j)+1}{2} & \text { for } j>i\end{cases} \\
& b_{i}=\frac{2(n-i)+1}{2}
\end{aligned}
$$

It should be noted that, in case of the rheonomic constraints, the transformation equations (1) contain time

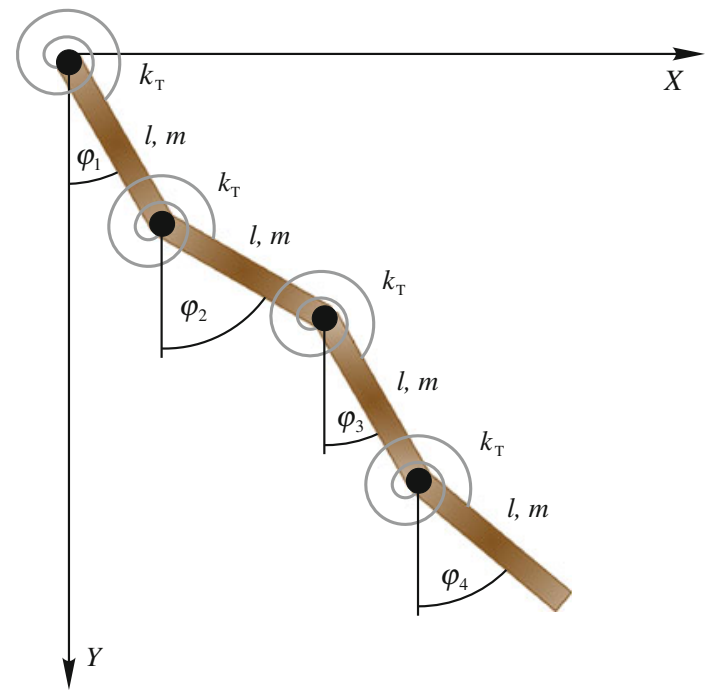

Fig. 1 A discrete model of a rope with elastic joints

explicitly, which affects the Lagrangian [and obviously the equations (2) too]. On the other hand, the well known condition for conservation of the energy $E=T+V$ by mechanical systems is:

$\frac{\partial L}{\partial t}=0$

Thus, the considered system is conservative, if only

$x_{0}(t) \equiv 0 \quad$ and $\quad y_{0}(t) \equiv 0$

which means that the constraints are scleronomic.

Off course, the presented model does not include any elasticity features. While the members are ideal rigid bodies preventing from deformation, the idealized links enable to bend the modelled rope unrestrictedly. In order to eliminate this disadvantage, we introduce bending stiffness to the model by using the spiral springs conception. More precisely, we assume that identical springs with stiffness $k_{T}$ are placed in each of the joints (also in the support) as shown in Fig. 1.

To gain an insight into the proposed model of transverse elasticity, consider a fragment of the imaginable rope in a local coordinate system $x y$ (see Fig. 2), where curvilinear coordinate is denoted by $s$. Assume that the curve has a radius $R$ at a point $P_{1}$. Let us perform a simple discretization of the rope by inscribing two adjacent rigid elements. If we specify the positions of the points $P_{0}, P_{1}$ and $P_{2}$ in the local coordinate system:

$$
\begin{aligned}
& x_{(0)}=0, \quad y_{(0)}=0 \\
& x_{(1)}=l \sin \varphi_{1}, \quad y_{(1)}=l \cos \varphi_{1} \\
& x_{(2)}=l\left(\sin \varphi_{1}+\sin \varphi_{2}\right), \quad y_{(2)}=l\left(\cos \varphi_{1}+\cos \varphi_{2}\right)
\end{aligned}
$$




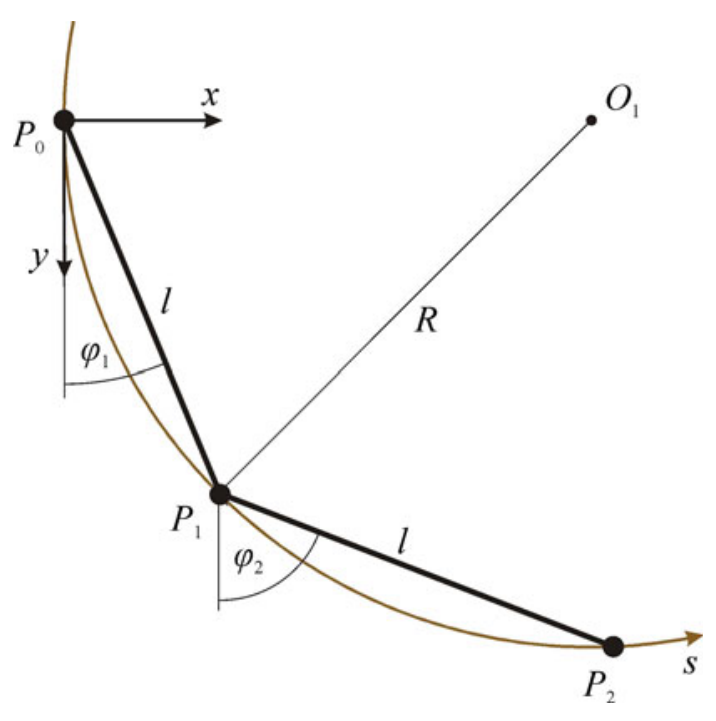

Fig. 2 Discretized fragment of the rope

and treat the coordinates as functions of the variable $s$, then we can easily approximate derivatives of $x(s)$ and $y(s)$ with respect to $s$ at $P_{1}$ by using the central difference schemes:

$x^{\prime} \approx \frac{x_{(2)}-x_{(0)}}{2 l}, \quad y^{\prime} \approx \frac{y_{(2)}-y_{(0)}}{2 l}$

$x^{\prime \prime} \approx \frac{x_{(2)}-2 x_{(1)}+x_{(0)}}{l^{2}}, \quad y^{\prime \prime} \approx \frac{y_{(2)}-2 y_{(1)}+y_{(0)}}{l^{2}}$

Next, we apply a formula for the radius of curvature for a plane curve given parametrically:

$R=\frac{\left(x^{\prime 2}+y^{\prime 2}\right)^{\frac{3}{2}}}{\left|\begin{array}{ll}x^{\prime} & y^{\prime} \\ x^{\prime \prime} & y^{\prime \prime}\end{array}\right|}$

Inserting (5) into (6) leads to

$R=-\frac{l}{2} \cos (\theta / 2) \cot (\theta / 2)$,

where $\theta=\varphi_{2}-\varphi_{1}$ is a relative generalized coordinate. Now we use a basic formula of strength of materials - the relation between the radius of curvature $R$ of a beam and the bending moment $M$ :

$\frac{1}{R}=\frac{M}{K}$

where $K$ is the flexural rigidity. Hence

$M=\frac{K}{R}=-\frac{2 K}{l} \frac{\tan (\theta / 2)}{\cos (\theta / 2)}$

A shape of the resulting nonlinear spring characteristic can be seen in Fig. 3. If one member of the system rotates through an angle of $\pm \pi$ with respect to the other segment, the elastic force $M$ grows infinitely, which prevents from an unnatural full rotation.

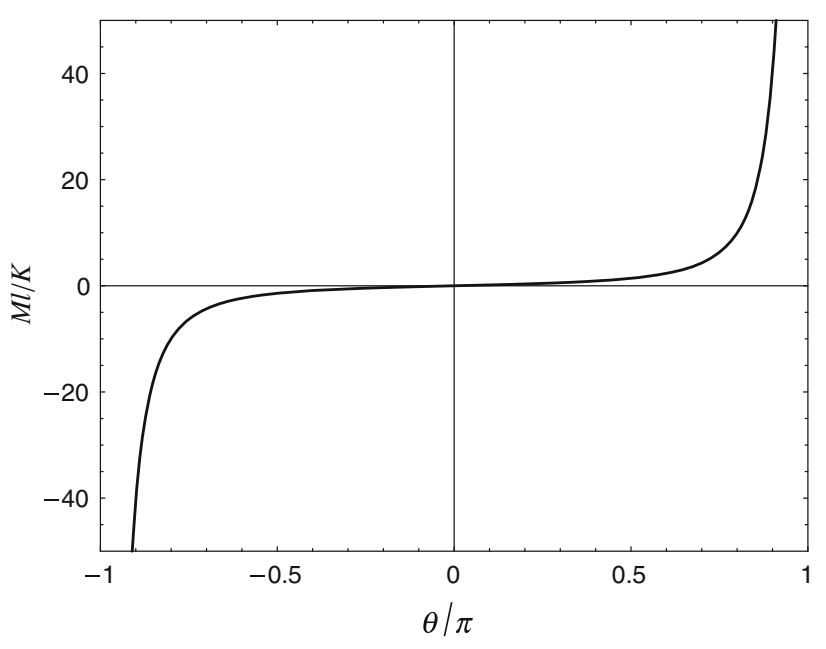

Fig. 3 The resulting nonlinear spring characteristic

To introduce the elasticity idea to the mathematical model represented by (2), we write a generalized formula for the moment $M$ associated with the $i$ th spring:

$M_{i}=-\frac{2 k_{T}}{l} \frac{\tan \left(\theta_{i} / 2\right)}{\cos \left(\theta_{i} / 2\right)}$

where $\theta_{i}$ is specified below

$\theta_{i}= \begin{cases}\varphi_{i} & \text { for } i=1 \\ \varphi_{i}-\varphi_{i-1} & \text { for } i=2,3, \ldots, n\end{cases}$

Now we consider the virtual work done by the moments of the forces in the joints:

$\delta W=M_{1} \delta \theta_{1}+M_{2} \delta \theta_{2}+\cdots+M_{n} \delta \theta_{n}$

Hence, the generalized forces resulting from the transverse elasticity and associated with the generalized coordinates $\varphi_{i}$ are

$Q_{i}^{T}= \begin{cases}M_{i}-M_{i+1} & \text { for } i=1,2, \ldots, n-1 \\ M_{i} & \text { for } i=n\end{cases}$

If we consider the Lagrange equations in the following form:

$\frac{d}{d t}\left(\frac{\partial T}{\partial \dot{q}_{i}}\right)-\frac{\partial T}{\partial q_{i}}=Q_{i}^{p}+Q_{i}^{n p}, \quad i=1,2, \ldots, n$,

where $Q_{i}^{p}$ and $Q_{i}^{n p}$ are generalized potential and nonpotential forces, respectively, then the former ones may be determined by combining the generalized forces generated by the gravity forces, expressed in (2) as

$Q_{i}^{G}=-m g l b_{i} \sin \varphi_{i}, \quad i=1,2, \ldots, n$

with the generalized forces (11). Still excluding the nonpotential forces $\left(Q_{i}^{n p}=0\right.$ for $\left.i=1,2, \ldots, n\right)$, one can obtain the final form of the equations of motion for the 
system with the elastic joints:

$$
\begin{aligned}
& \sum_{j=1}^{n} a_{i j} \ddot{\varphi}_{j} \cos \left(\varphi_{i}-\varphi_{j}\right)+\sum_{j=1}^{n} a_{i j} \dot{\varphi}_{j}^{2} \sin \left(\varphi_{i}-\varphi_{j}\right) \\
& \quad+\frac{b_{i}}{l}\left(\ddot{x}_{0} \cos \varphi_{i}-\ddot{y}_{0} \sin \varphi_{i}\right) \\
& =\frac{1}{m l^{2}}\left(Q_{i}^{G}+Q_{i}^{T}\right), \quad i=1,2, \ldots, n
\end{aligned}
$$

where the forces $Q_{i}^{T}$ and $Q_{i}^{G}$ are given by (11) and (12) respectively; the coefficients $a$ and $b$ are specified in (3).

\section{Numerical experiments}

On the basis of the mathematical model (13), we can easily come to a numerical description of the problem. Let us write the equations of motion in the concise form

$\mathbf{M}(\mathbf{q}) \ddot{\mathbf{q}}=\mathbf{f}(t, \mathbf{q}, \dot{\mathbf{q}})$

where the matrix $\mathbf{M}$ depends on the vector of dependent variables $\mathbf{q}$ and $t$ represents time as the independent variable. However, from the computational point of view the system (14) must be reformulated as a system of $2 n$ first-order differential equations

$\hat{\mathbf{M}}(\mathbf{x}) \dot{\mathbf{x}}=\hat{\mathbf{f}}(t, \mathbf{x})$

The modified matrix $\hat{\mathbf{M}}$, the modified vector $\hat{\mathbf{f}}$, and the vector $\mathbf{x}$ of unknown functions are structured as follows:

$\hat{\mathbf{M}}=\left[\begin{array}{cc}\mathbf{I} & \mathbf{0} \\ \mathbf{0} & \mathbf{M}\end{array}\right], \quad \hat{\mathbf{f}}=\left[\begin{array}{l}\mathbf{u} \\ \mathbf{f}\end{array}\right], \quad \mathbf{x}=\left[\begin{array}{l}\mathbf{q} \\ \mathbf{u}\end{array}\right]$

where I is an identity matrix and $\mathbf{u}$ is substituted for $\dot{\mathbf{q}}$. Finally, we write the initial value problem as

$$
\begin{aligned}
& {\left[\begin{array}{cc}
\mathbf{I} & \mathbf{0} \\
\mathbf{0} & \mathbf{M}
\end{array}\right]\left[\begin{array}{c}
\dot{\mathbf{q}} \\
\dot{\mathbf{u}}
\end{array}\right]=\left[\begin{array}{c}
\mathbf{u} \\
\mathbf{f}
\end{array}\right]} \\
& \mathbf{q}(0)=\mathbf{q}_{0}, \quad \mathbf{u}(0)=\mathbf{u}_{0}
\end{aligned}
$$

As can be seen above, the applied multi-body approach leads to the large system, whose size depends on density of discretization of the rope imagined as a continuum. What is more, it should be emphasized that the given problem is more complex than standard systems of ordinary differential equations (ODE) of the explicit form $\dot{\mathbf{x}}=\hat{\mathbf{f}}(t, \mathbf{x})$. In fact, the left-hand side matrix $\hat{\mathbf{M}}$ in (15) makes the equations coupled, which results in several derivatives in each ODE. Consequently, the given system is classified as implicit ODE or, more generally, as a system of differential/algebraic equations (DAE).

Generally speaking, DAEs require another approachdifferent from the fundamental and well-known numerical methods. Existing algorithms involve more sophisticated strategies than in case of explicit ODEs, especially when it comes to step-size control, method order control and error estimates. Some of the ideas are described in [9-12], whereas [13] exhibits several difficulties, which can occur when dealing with DAEs.

As with the problem analyzed in [7], we apply the MEBDFV solver designed by Abdulla and Cash of Imperial College, London (Department of Mathematics). Actually, the code is one of the few available suited for the solution of DAEs with non-constant matrix $\hat{\mathbf{M}}$, as in (15). The authors implement the modified extended backward differentiation formulas (MEBDF) developed by Cash (1980). Similarly to the BDF schemes, widely used for solving ODEs, the solution $\mathbf{x}_{i}$ at the current mesh point $t_{i}$ is approximated using the past values $\mathbf{x}_{i-1}, \mathbf{x}_{i-2}, \ldots, \mathbf{x}_{i-k}$. However, a key idea of MEBDF is to include the so called 'superfuture' value $\mathbf{x}_{i+1}$ in the same computation step. We included an outline of the method in [8]; more details are described in papers of Cash $[10,11,14]$.

Now let us turn to numerical experiments. The first one refers to a conservative scleronomic system, whereas the other example is connected to a system with rheonomic constraints.

\subsection{Experiment 1}

We consider a model of a total length $n l=1 \mathrm{~m}$ and total mass $n m=0.1 \mathrm{~kg}$, which consists of $n=20$ segments; the supposed spring constant $k_{T}=0.001 \mathrm{Nm}^{2}$. The initial configuration of the rope, with the position of the tip: $x=0.4$ $\mathrm{m}, y=0 \mathrm{~m}$, is specified by a catenary curve, whereas the generalized velocities are assumed to be equal to zero at a start point: $\dot{\varphi}_{i}(0)=0$ for $i=1,2, \ldots, n$. Both the functions $x_{0}(t)$ and $y_{0}(t)$ are as follows:

$x_{0}(t) \equiv 0$ and $y_{0}(t) \equiv 0$

In the rigid multi-body system (see [7]), a simple construction of the model has enabled the segments to experience full rotations around the joints. Especially the last member, as the least constrained one, has rotated rapidly in an unnatural manner. Distinct symptoms of such behaviour can be seen in Fig. 4: high differences between orientations of the two last members $\left(\varphi_{20}\right.$ and $\left.\varphi_{19}\right)$ and rapid changes of the function $\varphi_{20}(t)$.

On the contrary, in case of the elastic joints, the springs are supposed to block the strange and sudden rotations. In fact, the functions $\varphi_{20}(t)$ and $\varphi_{19}(t)$ do not differ considerably (see Fig. 5), which can be evaluated via the maximum norm:

$\max _{0<t<5}\left|\varphi_{20}(t)-\varphi_{19}(t)\right|=1.415$ 


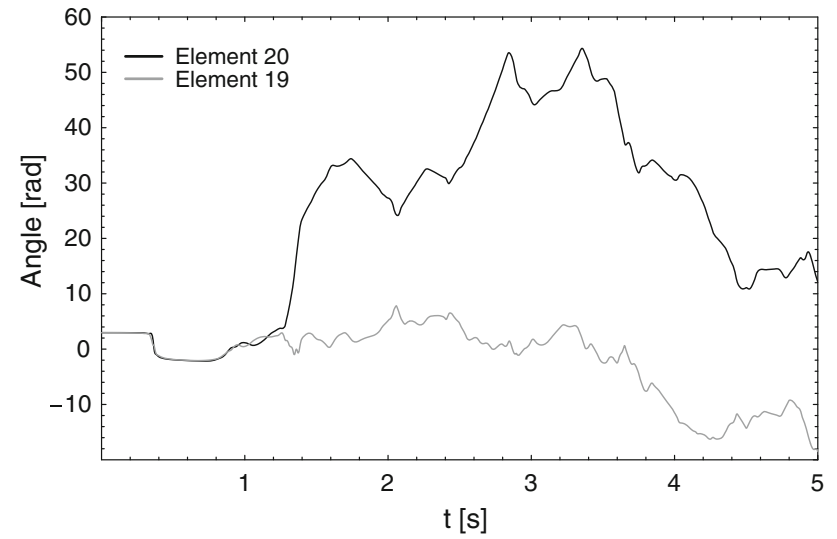

Fig. 4 Orientations of the two last elements (19 and 20) in time for the inelastic model; a problem with parameters and initial conditions as in Sect. 3.1

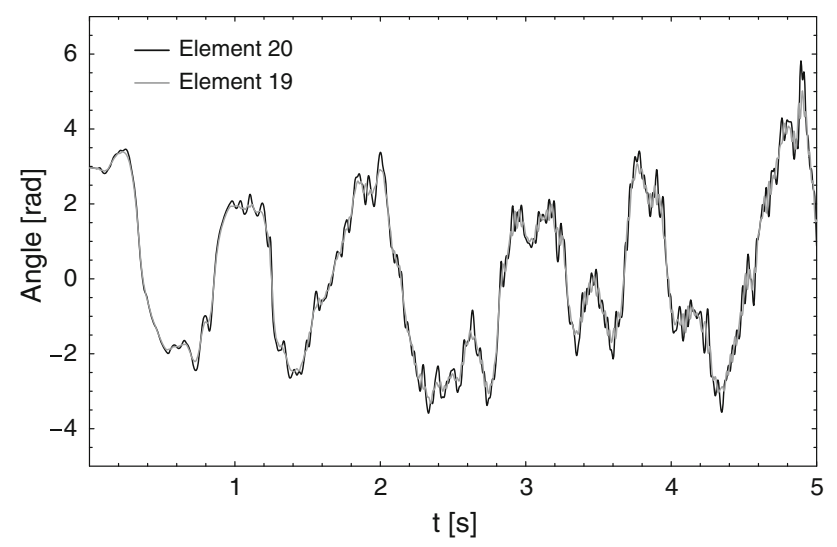

Fig. 5 Orientations of the two last elements (19 and 20) in time for the model with elastic joints; results obtained in Sect. 3.1

Indeed, the simulated motion seems to be smoother and does not exhibit the effects, which are difficult to imagine in a real plane motion.

However, to analyze how the elasticity actually affects the dynamics, let us look at the system globally, by mean of its energy. Obviously, the kinetic energy $T$ contains an additional component resulting from the elastic work, which determines the structure of $T$. More precisely, if we distinguish two components of the kinetic energy-the translational and rotational:

$T=T_{t}+T_{r}$

then we can evaluate their proportions. Let us concentrate on the discrete functions $T\left(t_{i}\right)$ and $T_{r}\left(t_{i}\right)$ where $i=0,1, \ldots, N$ and $N$ is a given number of time-steps. The average values

$\bar{T}=\frac{1}{N} \sum_{i=0}^{N} T\left(t_{i}\right), \quad \bar{T}_{r}=\frac{1}{N} \sum_{i=0}^{N} T_{r}\left(t_{i}\right)$
Table 1 Contribution of the rotational kinetic energy $T_{r}$ to the total kinetic energy $T$ for problems with different stiffness $k_{T}$. Results obtained with time-step $\Delta t=1.0 \mathrm{E}-03 \mathrm{~s}$ for motion lasting $t=5 \mathrm{~s}$

\begin{tabular}{ll}
\hline$k_{T}\left(\mathrm{Nm}^{2}\right)$ & $\bar{T}_{r} / \bar{T}$ \\
\hline Rigid system & 0.085 \\
0.001 & 0.042 \\
0.002 & 0.062 \\
0.005 & 0.075 \\
0.010 & 0.081 \\
\hline
\end{tabular}

may serve as global measures of the functions. Performing simulations starting from the discussed initial conditions, for models with the same parameters but various stiffness $k_{T}$, we find that the contribution of the rotational kinetic energy increases with $k_{T}$, as presented in Table 1 . Thus, on the one hand, the spring in the joints eliminate the specific full rotations. On the other hand, after a short period of regular motion some individual, non-regular rotations appear (see Fig. 6) and their growing intensity depends strictly on the constant $k_{T}$. Beyond any doubt, the stabilizing role of the moments $M_{i}$ manifest itself in keeping relatively small differences between orientations of the adjacent members. Consequently, it leads to some rotational oscillations in a narrow range of angles with frequency depending on the springs stiffness.

As presented in Figs. 6 and 7, the oscillatory behaviour of the elements is reflected in both the kinetic and the potential energy of the mechanical system. In any case, one can easily test a fulfilment of the energy conservation principle, theoretically relevant to the considered example. In fact, the approximately straight line of the total energy $E=T+V$ includes some small fluctuations. In order to assess a quality of the solution, a relative error and its average value are calculated as follows:

$e_{R}\left(t_{i}\right)=\left|\frac{E\left(t_{i}\right)-E\left(t_{0}\right)}{E\left(t_{0}\right)}\right|$

$\bar{e}_{R}=\frac{1}{N} \sum_{i=0}^{N} e_{R}\left(t_{i}\right)$

Figure 8 shows the time dependence of the error (18); its average value for the presented time interval equals $\bar{e}_{R}=$ $2.8 \mathrm{E}-06$.

Finally, let us consider the problem of disretization of the rope, which was outlined in [7]. Theoretically, the multibody model is increasingly better representation of the physical system as $n$ tends to infinity while $l$ tends to zero. Thus, there should be convergence of solutions for gradually higher discretization density. However, comparing two numerical solutions obtained for different values of $n$ is not so trivial. Let $n_{1}$ and $n_{2}$ be arbitrary numbers of elements. The question 

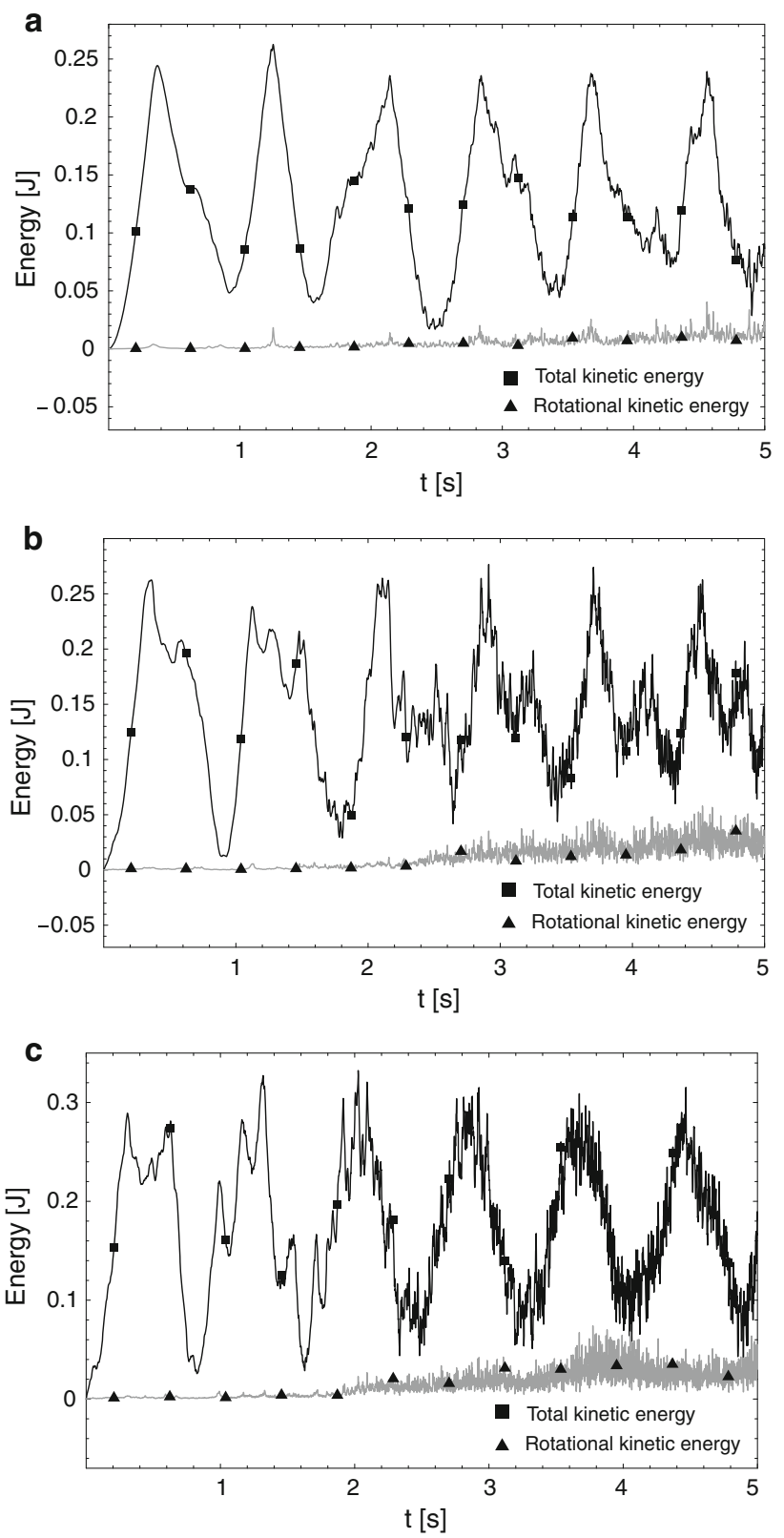

Fig. 6 Total kinetic energy and rotational kinetic energy of the system: $\mathbf{a} k_{T}=0.001\left(\mathrm{Nm}^{2}\right), \mathbf{b} k_{T}=0.005\left(\mathrm{Nm}^{2}\right), \mathbf{c} k_{T}=0.010\left(\mathrm{Nm}^{2}\right)$; after a short period of smooth dynamics, specific oscillatory rotations appear

is how to measure a distance

$d_{n_{1}, n_{2}}=\left\|\mathbf{q}_{n_{1}}-\mathbf{q}_{n_{2}}\right\|$

between the solutions $\mathbf{q}_{n_{1}}$ and $\mathbf{q}_{n_{2}}$ given for $n=n_{1}$ and $n=n_{2}$, respectively.

In this paper we propose the following simple algorithm. First, we choose a set of $n^{*}$ control points $\left\{\left(x_{j}^{*}, y_{j}^{*}\right)\right\}_{j=1}^{n^{*}}$ distributed along the rope uniformly: from its fixed end to the other one. Now, using geometrical distances between the corresponding points in the both cases, one can form the

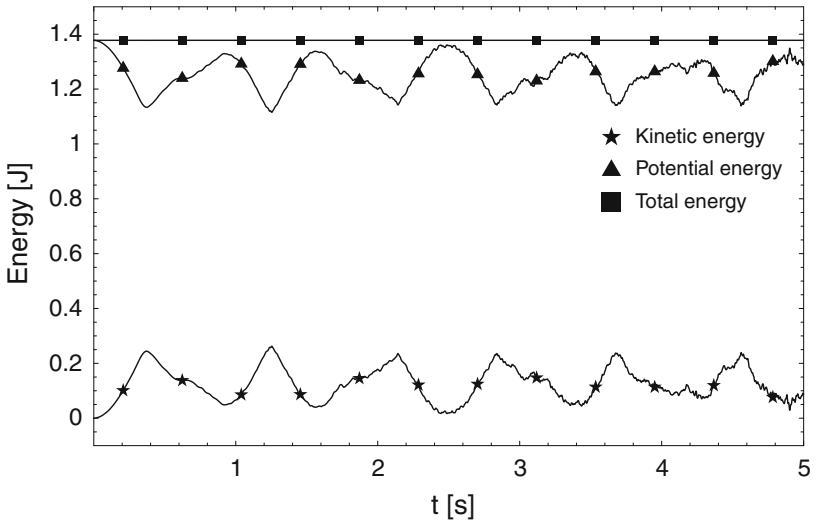

Fig. 7 Kinetic, potential and total energy of the system; the energy conservation principle approximately fulfilled

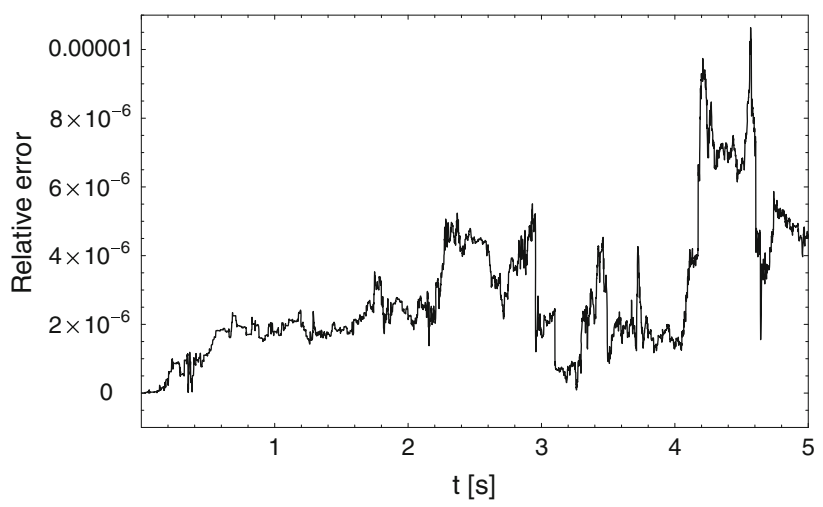

Fig. 8 Relative error of the total energy of the system

following norm:

$d_{n_{1}, n_{2}}=\sqrt{\frac{1}{n^{*}} \sum_{j=1}^{n^{*}}\left[\left(x_{j, n_{1}}^{*}-x_{j, n_{2}}^{*}\right)^{2}+\left(y_{j, n_{1}}^{*}-y_{j, n_{2}}^{*}\right)^{2}\right]}$,

where $x_{j, n_{1}}^{*}, y_{j, n_{1}}^{*}$ and $x_{j, n_{2}}^{*}, y_{j, n_{2}}^{*}$ denote coordinates of the controlled points calculated from the solution vectors $\mathbf{q}_{n_{1}}$ and $\mathbf{q}_{n_{2}}$, respectively.

In this example, we examine the distance variation with time for three pairs of solutions: $d_{20,25}, d_{30,35}, d_{40,45}$. Although the difference $\left(n_{2}-n_{1}\right)$ is kept constant, value of the distance is expected to decrease for the consecutive cases. Figure 9 presents the results obtained for $n^{*}=51$; the model parameters are assumed as before $\left(k_{T}=0.001 \mathrm{Nm}^{2}\right)$. Generally, the functions $d_{n_{1}, n_{2}}(t)$ grows over time, which may be an effect of the system oscillatory behaviour. However, the solution distance reaches lower values as the discretization density is raised. We are convinced that the solution convergence could be clearly greater, if only there was dissipation included in the model. 

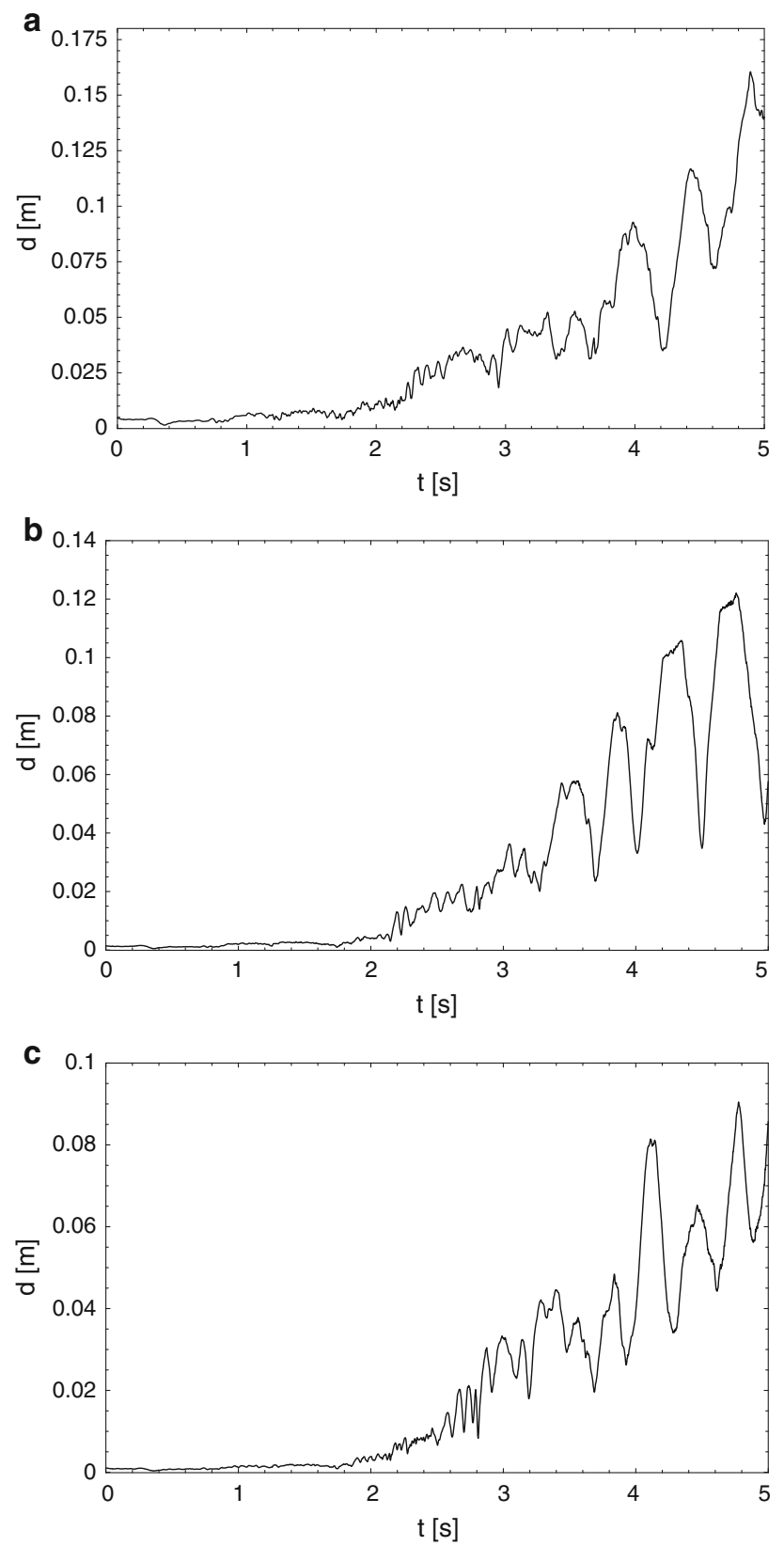

Fig. 9 Distance between solutions: a $n_{1}=20$ and $n_{2}=25$, b $n_{1}=30$ and $n_{2}=35$, c $n_{1}=40$ and $n_{2}=45$. Results obtained for $n^{*}=51$

\subsection{Experiment 2}

Now we turn to a case including rheonomic constraints. Let us consider a system with the same total mass $\mathrm{nm}$ and total length $n l$ but different number of members $n=30$ and the stiffness $k_{T}=0.002 \mathrm{Nm}^{2}$. Initial conditions of motion are

$\varphi_{i}(0)=0, \quad \dot{\varphi}_{i}(0)=0, \quad i=1,2, \ldots, n$

so that the rope hangs down until motion of the support in the horizontal direction is enforced according to the

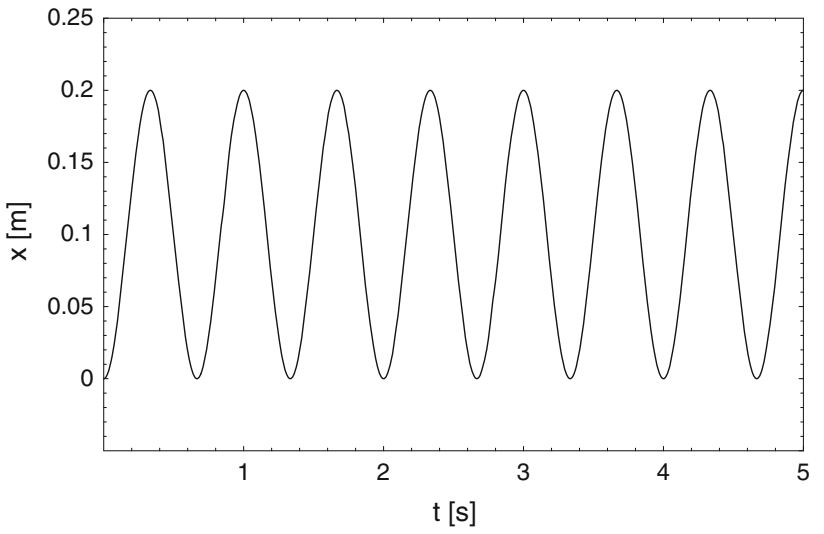

Fig. 10 Constraints function $x_{0}(t)$ : the support performs periodical manoeuvres

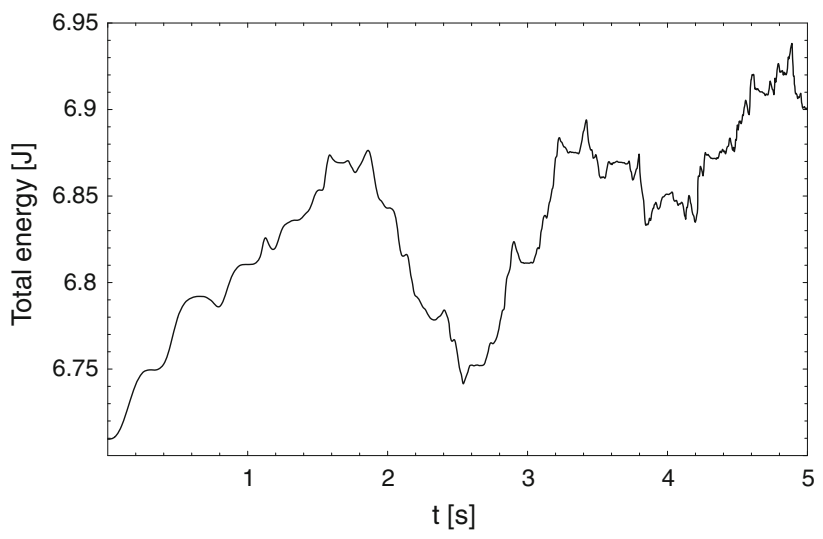

Fig. 11 Total energy of the non-conservative system

following function:

$x_{0}(t)=A \sin ^{2}(\pi B t)$

where $A$ and $B$ are constants; here we take $A=0.2 \mathrm{~m}$ and $B=1.5 \mathrm{~s}^{-1}$. It is easy to notice, that the support reciprocates, activating the entire system periodically (see Fig. 10).

In this example let us focus on the results verification, which turns out to be more complex than before, because the given system is non-conservative. Thus, it is not enough to analyze its total energy $E$, which varies with time as shown in Fig. 11. In view of this, we use the work-energy relation:

$\Delta T=W$

Accordingly, the change in the kinetic energy of the system is equal to the work $W$ done by all forces during the same actual displacements. In particular, if $T(0)=0$, then $\Delta T=T$. However, to apply (22) properly, we shall look at the dynamical system in a slightly different manner. Firstly, the potential generalized forces resulting from the model (13) are given by

$Q_{i}^{p}=Q_{i}^{T}+Q_{i}^{G}, \quad i=1,2, \ldots, n$ 


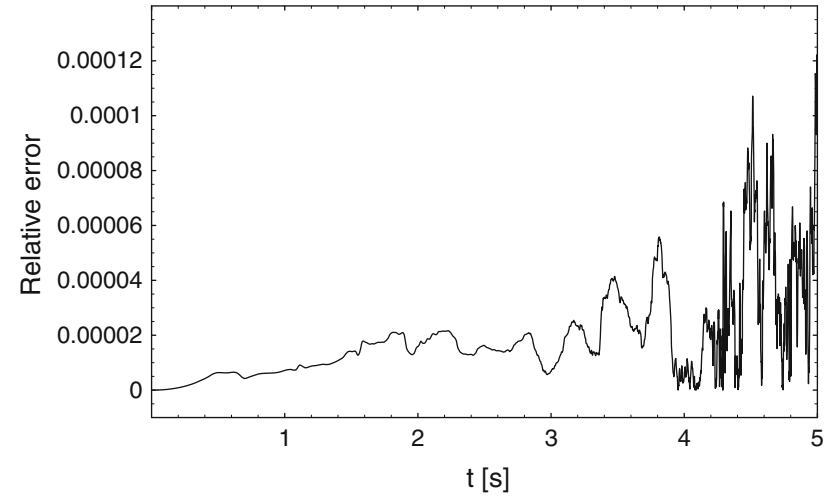

Fig. 12 Relative error of the difference between kinetic energy and work of all forces

Secondly, we take into account the components in (13), which come strictly from the rheonomic constraints, and treat them as the generalized non-potential forces:

$Q_{i}^{n p}=-m l b_{i}\left(\ddot{x}_{0} \cos \varphi_{i}-\ddot{y}_{0} \sin \varphi_{i}\right), \quad i=1,2, \ldots, n$

Now, let $T^{*}$ be the kinetic energy of the system in the reference frame moving with the support point $O$. In other words, $T^{*}$ is unaffected by a translation of the entire system in space, so that $T^{*}$ is formed by neglecting the components of $T$ which contain time explicitly. Finally, we can rewrite the relationship (22) in the following form:

$T^{*}=\sum_{i=1}^{n} \int_{S} Q_{i} d \varphi_{i}$

where $S$ is the given configuration path and the work $W$ is expressed with use of the generalized forces:

$Q_{i}=Q_{i}^{p}+Q_{i}^{n p}$

Specifying an absolute error as a difference between the lefthand side and the right-hand side of (23), one may determine a relative error as follows:

$e_{R}\left(t_{i}\right)=\left|\frac{T^{*}\left(t_{i}\right)-W\left(t_{i}\right)}{\max \left(T^{*}\right)}\right|$

As shown in Fig. 12, the error in the time interval $0 \leq t \leq 5 \mathrm{~s}$ does not exceed 1.3E-04. Its average value, computed according to formula (19) with stepsize $\Delta t=$ $1.0 \mathrm{E}-03 \mathrm{~s}$, equals $\bar{e}_{R}=1.9 \mathrm{E}-05$.

\section{Conclusions}

In this paper we have considered the discrete model of a rope, whose rigid members are linked by so called elastic joints. More precisely, the transverse elasticity has been modeled by means of the classical bending conception (simple theory of elastic bending) and basic geometrical relations. This has led to the non-linear characteristic of spiral springs placed in the joints. Such an elastic characteristic is typical for hard springs [5] and close to the intuitive view on behaviour of folded ropes or cables.

The Lagrange equations of motion have been derived for the general case of the system with rheonomic constraints. The elasticity idea has been introduced to the description by specifying the potential generalized forces in the joints. In comparison with the non-elastic system presented in [7], the obtained mathematical model is not much more complicated, in spite of the additional nonlinearity. In view of computational methods, the resulting problem is classified as a system of DAEs, typical for multi-body systems dynamics.

The applied MEBDFV code is one of the most efficient techniques designed for solving such complex problems. In numerical experiments, validity of the results has been examined in terms of energy principles. In case of the conservative scleronomic system, energy conservation has been observed. On the other hand, the solution for the non-conservative system has met the work-energy relation. In the both cases, very low values of the relative error exclude numerical dissipation.

The performed simulations also allow us to assess the model modification. Compared with the multiple physical pendulum (see [7]), the transverse elasticity eliminates the full rotations of the particular segments-the bahaviour which actually is unnatural for a real plane motion of dynamical systems. However, contribution of the rotational kinetic energy to the total kinetic energy increases with the stiffness $k_{T}$. Consequently, the chaotic-looking dynamics does not become weakened due to some specific rotational oscillations driven by the stabilizing activity of the springs. Presumably, the model needs to be developed by replacing the elastic springs with the viscoelastic ones, which could damp out the transverse oscillations.

All in all, the considered problem exhibits powerful capabilities of the approach based on analytical mechanics and commonly available high-performance numerical solver. The promising results encourage us to consider more realistic models of a rope, with special reference to air resistance and chaotic dynamics analysis.

Open Access This article is distributed under the terms of the Creative Commons Attribution Noncommercial License which permits any noncommercial use, distribution, and reproduction in any medium, provided the original author(s) and source are credited.

\section{References}

1. Wong C, Yasu K (2006) Falling chains. Phys Rev Lett 74(6): 490-496

2. Pierański P, Tomaszewski W (2005) Dynamics of ropes and chains: I. The fall of the folded chain. New J Phys 7(45)

3. Goriely A, McMillen T (2002) Shape of a cracking whip. Phys Rev Lett $88(24)$ 
4. Gatti C, Perkins N (2002) Physical and numerical modeling of the dynamic behavior of a fly line. J Sound Vib 255(3):555-577

5. Lobas L (2005) Generalized mathematical model of an inverted multilink pendulum with follower force. Int Appl Mech 41(5): 566-572

6. Kawaji S, Kanazawa K (1991) Control of double inverted pendulum with elastic joint. IEEE/RSJ International Workshop on Intelligent Robots and Systems '91, vol 2, pp 946-951

7. Fritzkowski P, Kamiński H (2008) Dynamics of a rope as a rigid multibody system. J Mech Mater Struct 3(6):1059-1075

8. Fritzkowski P, Kamiński H (2009) Dynamics of a rope modeled as a discrete system with extensible members. Comput Mech 44(4):473-480

9. Gear CW (2006) Towards explicit methods for differential algebraic equations. BIT Numer Math 46:505-514
10. Cash JR (2003) Efficient numerical methods for the solution of stiff initial-value problems and differential algebraic equations. Proc $\mathrm{R}$ Soc Lond A 459:797-815

11. Cash JR, Considine S (1992) An MEBDF Code for Stiff Initial Value Problems. ACM Trans Math Softw 18(2):142-155

12. Cash JR (2000) Modifed extended backward differentiation formulae for the numerical solution of stiff initial value problems in ODEs and DAEs. J Comput Appl Math 125:117-130

13. Petzold L (1982) Differential/algebraic equations are not ODEs. SIAM J Sci Stat Comput 3(3):367-384

14. Abdulla TJ, Cash JR, Diamantakis MT (2001) An MEBDF package for the numerical solution of large sparse systems of stiff initial value problems. Comput Math Appl 42:121-129 